\title{
A heart valve is no stronger than its weakest link: The need to improve durability of pericardial leaflets
}

\author{
Muralidhar Padala, $\mathrm{PhD}$
}

\footnotetext{
From the Structural Heart Research \& Innovation Laboratory, Division of Cardiothoracic Surgery, Carlyle Fraser Heart Center, Emory University, Atlanta, Ga.

Disclosures: Author has nothing to disclose with regard to commercial support.

Received for publication March 1, 2018; accepted for publication March 7, 2018; available ahead of print April 7, 2018.

Address for reprints: Muralidhar Padala, PhD, Structural Heart Research \& Innovation Laboratory, Division of Cardiothoracic Surgery, Carlyle Fraser Heart Center, Emory University, 380B Northyards Blvd, Atlanta, GA 30313 (E-mail: spadala@emory.edu).

J Thorac Cardiovasc Surg 2018;156:207-8

$0022-5223 / \$ 36.00$

Copyright (c) 2018 by The American Association for Thoracic Surgery

https://doi.org/10.1016/j.jtcvs.2018.03.034
}

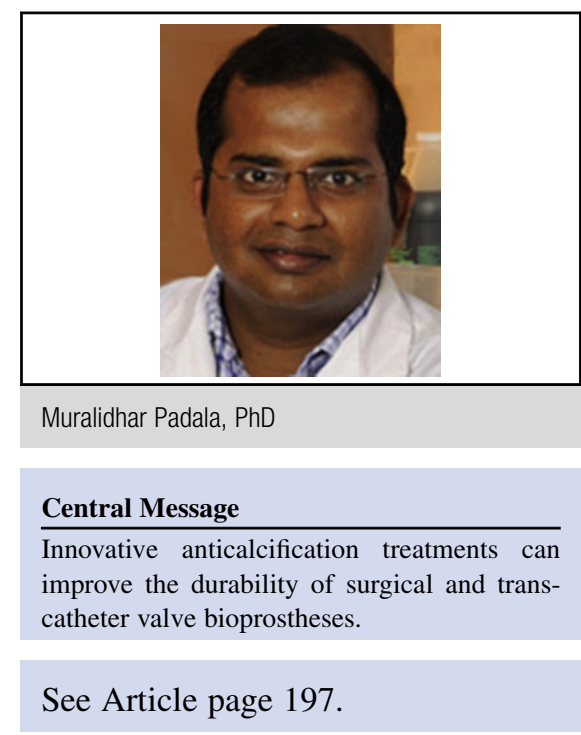

The durability of bioprosthetic heart valves continues to increase with each decade, with improved patient selection, better protocols for tissue fixation, and the availability of various anticalcification treatments. Despite these encouraging outcomes, calcification of bioprosthetic valves continues to remain a challenge, with reoperation rates at 10 years after implantation ranging from $3 \%$ to $20 \%{ }^{1,2}$ The advent of transcatheter aortic valve replacement (TAVR) and the ability to perform valve-in-valve replacements has shifted focus slightly from investing in strategies to improve material durability toward an approach to develop low-profile valves that can be implanted into the failing original bioprosthetic valve. Although this is an alluring idea, the fact that TAVR valves are manufactured from thinner leaflets of the same material indicates the potential for these secondary replacements to calcify and deteriorate with time. This risk becomes higher as TAVR valves are used in patients at lower risk with several decades of expected survival after the procedure. Dvir and colleagues ${ }^{3}$ studied 378 patients who received a TAVR valve and observed a $50 \%$ structural valve deterioration rate between 5 and 7 years after the implantation.

Calcification of bioprosthetic heart valves could occur from external patient-specific factors (eg, hyperlipidemia, chronic kidney disease, young age) or from internal valve material-specific factors (eg cellular remnants, calcium entrenchment into phospholipids in the cellular remnants, traces of free glutaraldehyde), or from unknown interactions between both types of factor. In their publication in this issue of the Journal, Meuris and colleagues ${ }^{4}$ report the efficacy of the LivaNova FREE treatment (LivaNova PLC, London, United Kingdom), which involves octanediol-ethanol to remove phospholipids in the cellular remnants, a taurine wash to neutralize unbound aldehydes left after glutaraldehyde fixation, and storage in an aldehyde-free solution containing preservatives such as parabens. The logic behind this treatment strategy is sound. Plasma membranes and membrane organelles in the cellular remnants of the bovine pericardium are rich in phospholipids, which are a source of phosphorus that favors calcium phosphate crystallization in the tissue. Vyavahare and associates $^{5}$ pioneered a high-concentration ethanol (a short chain alcohol) anticalcification treatment for bovine pericardium to remove phospholipids ${ }^{5}$; others have extended this to long-chain alcohols like octanediol, which are structurally like phospholipids ${ }^{6}$ Because the solubility of octanediol in water is limited, buffered ethanolic solution improves solubility and tissue penetrance. Pathak and associates $^{7}$ demonstrated the benefit of such a treatment in reducing tissue calcification, with possibly better outcomes with use of octanediol in an ethanol solution than with ethanol alone. Meuris and colleagues ${ }^{4}$ used this technique reported by Pathak and associates, ${ }^{7}$ and as such are not innovative in this regard. In the next step, Meuris and colleagues ${ }^{4}$ detoxify the tissue with taurine (also known as aminoethane sulfonic acid), which has an amine group $\left(\mathrm{NH}_{2}\right)$ to bond with the aldehyde group of any unconjugated glutaraldehyde left after tissue fixation. It is possible that the combination of phospholipid repletion and subsequent treatment with taurine, may allow better penetration of the taurine into the tissue and aid in better outcomes. Finally, the tissue is preserved in aldehyde-free solution with parabens, which is a common preservative used in foods and cosmetic products. By addressing these different components contributing to calcification, the 
FREE treatment is theoretically an effective strategy to improve durability of bioprosthetic heart valves.

The results of Meuris and colleagues, ${ }^{4}$ however, fall short in validating this theoretic excitement, as I shall elucidate. The phospholipid content in the tissue treated with FREE treatment was equivalent to that of Linx ( $>60 \%$ volume ethanol treatment to remove phospholipids, marketed by St Jude Medical and used in Trifecta [St Jude Medical Inc, St Paul, Minn]) and Thermafix (heat treatment to denature proteins and phospholipids, marketed by Edwards Lifesciences and used in Perimount [Irvine, Calif]). This observation makes one wonder whether the theoretic benefit of treating with long-chain octanediol was realized at all, because treatment with ethanol alone (Linx) seems to have the same effect. Extractable aldehydes were significantly lower in the FREE treatment compared to tissues treated with Linx or Thermafix, but at day 6 the levels were comparable between Thermafix and FREE. The calcium content after subcutaneous implantation in rats were equivalent among the 3 treatments, and the preclinical implants are too underpowered to deduce any conclusions. Bringing together these results, it would be reasonable to deduce that the FREE treatment may be noninferior to Linx and Thermafix, but for reasons unknown the theoretic benefits laid out from FREE treatment relative to other treatments are lost in translation.

Despite the theoretic benefits and noninferiority of the FREE treatment strategy, the longer term outcomes in human patients can only be assessed with longitudinal observational studies. Most anticalcification strategies have succeeded in the subcutaneous rat and juvenile sheep models, but those favorable results could not be replicated in human patients, probably because of individual patient risk factors. Research to improve tissue durability should therefore continue, and probably accelerate, because of the rapid proliferation of TAVR. Ultimately, lack of tissue durability is the weakest link, whether a valve is deployed surgically or through a catheter, and the current fervor surrounding the delivery mode should not mask research in this area. I therefore congratulate Meuris and colleagues ${ }^{4}$ for their study.

\section{References}

1. Bourguignon T, El Khoury R, Candolfi P, Loardi C, Mirza A, Boulanger-Lothion J, et al. Very long-term outcomes of the Perimount aortic valve in patients aged 60 or younger. Ann Thorac Surg. 2015;100:853-9.

2. Forcillo J, Pellerin M, Perrault LP, Cartier R, Bouchard D, Demers P, et al. Carpentier-Edwards pericardial valve in the aortic position: 25-years experience. Ann Thorac Surg. 2013;96:486-93.

3. Dvir D. First look at long-term durability of transcatheter heart valves: assessment of valve function up to 10 years after implantation. Presented at: EuroPCR 2016; May 17-20, 2016; Paris, France.

4. Meuris B, De Praetere H, Strasly M, Trabucco P, Lai JC, Verbrugghe P, et al. A novel tissue treatment to reduce mineralization of bovine pericardial heart valves. J Thorac Cardiovasc Surg. 2018;156:197-206.

5. Vyavahare NR, Hirsch D, Lerner E, Baskin JZ, Zand R, Schoen FJ, et al. Prevention of calcification of glutaraldehyde-crosslinked porcine aortic cusps by ethanol preincubation: mechanistic studies of protein structure and water-biomaterial relationships. J Biomed Mater Res. 1998;40:577-85.

6. Pettenazzo E, Valente M, Thiene G. Octanediol treatment of glutaraldehyde fixed bovine pericardium: evidence of anticalcification efficacy in the subcutaneous rat model. Eur J Cardiothorac Surg. 2008;34:418-22.

7. Pathak CP, Adams AK, Simpson T, Phillips RE Jr, Moore MA. Treatment of bioprosthetic heart valve tissue with long chain alcohol solution to lower calcification potential. J Biomed Mater Res A. 2004;69:140-4. 